\title{
Deep Brain Stimulation in Trigeminal Autonomic Cephalalgias
}

\author{
Massimo Leone, ${ }^{*}$ Angelo Franzini, ${ }^{\dagger}$ Alberto Proietti Cecchini, ${ }^{*}$ Eliana Mea, ${ }^{*}$ \\ Giovanni Broggi, ${ }^{\dagger}$ and Gennaro Bussone* \\ *Headache Center, Neuromodulation Unit, Department of Neurology, and 'Department of Neurosurgery, Fondazione I.R.C.C.S. \\ Istituto Neurologico Carlo Besta, via Celoria 11, 20133 Milan, Italy
}

\begin{abstract}
Summary: Cluster headache $(\mathrm{CH})$, paroxysmal hemicrania $(\mathrm{PH})$, and short-lasting unilateral neuralgiform headache attacks with conjunctival injection and tearing (SUNCT syndrome) are primary headaches grouped together as trigeminal autonomic cephalalgias (TACs). All are characterized by short-lived unilateral head pain attacks associated with oculofacial autonomic phenomena. Neuroimaging studies have demonstrated that the posterior hypothalamus is activated during attacks, implicating hypothalamic hyperactivity in TAC pathophysiology and suggesting stimulation of the ipsilateral posterior hypothalamus as a means of preventing intractable $\mathrm{CH}$. After almost 10 years of experience, hypothalamic stimulation has proved successful in preventing pain attacks in approximately $60 \%$ of the 58 documented chronic drug-resistant $\mathrm{CH}$ patients implanted at various centers. Positive results have also been reported in drug-resistant SUNCT and PH. Microrecording studies on hypothalamic neurons are increasingly being performed and promise to make it possible to more precisely identify the target site. The implan-
\end{abstract}

tation procedure has generally proved safe, although it carries a small risk of brain hemorrhage. Long-term stimulation is proving to be safe: studies on patients under continuous hypothalamic stimulation have identified nonsymptomatic impairment of orthostatic adaptation as the only noteworthy change. Studies on pain threshold in chronically stimulated patients show increased threshold for cold pain in the distribution of the first trigeminal branch ipsilateral to stimulation. When the stimulator is switched off, changes in sensory and pain thresholds do not occur immediately, indicating that long-term hypothalamic stimulation is necessary to produce sensory and nociceptive changes, as also indicated by clinical experience that $\mathrm{CH}$ attacks are brought under control only after weeks of stimulation. Infection, transient loss of consciousness, and micturition syncope have been reported, but treatment interruption usually is not required. Key Words: Cluster headache, paroxysmal hemicrania, SUNCT syndrome, trigeminal autonomic cephalgias, hypothalamus, deep brain stimulation.

\section{INTRODUCTION}

Deep brain stimulation (DBS) is a therapeutic modality widely used to relieve conditions that are intractable to medications, including such conditions as movement disorders, epilepsy, and chronic pain. ${ }^{1}$ For relieving pain, the main targets are the periventricular and periaqueductal gray matter, sensory thalamus, and internal capsule, although results are variable. ${ }^{2}$ Both opioid and nonopioid mechanisms seem to be involved in DBS-induced analgesia. $^{3,4}$

Evidence of a physiological connection between headand-face sensitivity and specific brain areas involved in pain processing accumulated slowly during the second

Address correspondence and reprint requests to: Massimo Leone, Headache Center, Neuromodulation and Neurological Department, Fondazione Istituto Neurologico Carlo Besta, via Celoria 11, 20133 Milan, Italy. E-mail: leone@ istituto-besta.it. half of the 20th century. Sano et al..$^{5}$ found that facial stimulation could cause neuronal discharges in the posteromedial hypothalamus (picked up by an electrode), and Raskin et al. ${ }^{6}$ provided evidence of an efferent connection: $9 \%$ of patients (15 of 175) who underwent DBS for intractable low back pain developed new headache syndromes. The headaches were severe, with migrainous features, and were long-lasting ( 2 months to 10 years); in 11 cases, the electrode had been inserted into the periaqueductal gray. Veloso et al. ${ }^{3}$ also reported the development of de novo chronic headache in 15 of 64 patients $(23 \%)$ stimulated in the periaqueductal area, sensory thalamus, or internal capsule; there was generally a lag of approximately 2 months between implantation and headache onset, suggesting that prolonged stimulation was necessary to affect pain-related neural pathways or to change thresholds. More recently, Bittar et al. ${ }^{7}$ described a patient in whom stimulation of various parts of the periventricular region evoked somatopically reported re- 
sponses to all body areas, including the face and scalp. Taken together, findings from these studies raise the possibility that stimulation of specific brain areas may interfere with pain processing. ${ }^{8}$

\section{RATIONALE FOR HYPOTHALAMIC STIMULATION IN HEADACHE}

Neuroimaging findings both during and apart from headache attacks have provided the rationale for hypothalamic stimulation in headache, as well as important information on attack-related events and clues to pathophysiological mechanisms. ${ }^{9}$ Headaches studied by neuroimaging include migraine with and without aura, cluster headache, SUNCT syndrome (i.e., short-lasting unilateral neuralgiform headache attacks with conjunctival injection and tearing), hemicrania continua, and paroxysmal hemicrania $(\mathrm{PH}){ }^{9}$

Cluster headache, SUNCT, and PH are trigeminal autonomic cephalalgias characterized by brief severe pain attacks in the area of the first branch of the trigeminal nerve (15-180 minutes in cluster headache; 5-240 seconds in SUNCT; 2-30 minutes in PH), accompanied by ipsilateral oculofacial autonomic symptoms. ${ }^{10}$ During cluster headache attacks, PET imaging revealed activation of areas associated with pain modulation and perception (anterior cingulate cortex, insulae, and contralateral thalamus), but also activation of the posterior inferior hypothalamic gray matter ipsilateral to the pain. ${ }^{11}$ This latter area was initially thought to be activated only in cluster headache, and was proposed as the cluster generator. ${ }^{11}$ It was subsequently shown, however, that this hypothalamic area is also activated during SUNCT, ${ }^{12} \mathrm{PH},{ }^{13}$ and hemicrania continua. ${ }^{14}$ Hemicrania continua is a strictly unilateral headache accompanied by ipsilateral oculofacial autonomic symptoms not now considered to be a trigeminal autonomic cephalalgia, because of its long-lasting pain. ${ }^{10}$ Activation of the posterior hypothalamus seems to be specific to trigeminal autonomic cephalalgias and hemicrania continua; it does not occur in migraine or experimentally induced trigeminal pain. ${ }^{9}$

In 1999, a voxel-based morphometry study demonstrated increased neuronal density in the posterior inferior hypothalamic gray matter in cluster headache patients in remission phase. ${ }^{15}$ This was the first identification of a lesion apparently associated with a primary headache.

These neuroimaging data therefore suggested that stereotactic stimulation targeting the hypothalamus might be a useful new therapeutic approach to chronic intractable forms of cluster headache. ${ }^{16}$ New therapeutic approaches were needed, given that $15-20 \%$ of cluster headache patients experience chronic cluster headache, and some of these do not respond to drugs. ${ }^{17,18}$ Such patients are a major clinical problem because of the severity and frequency (several times a day) of their headache attacks. Destructive surgery that interrupts the trigeminal sensory or autonomic (cranial parasympathetic) pathway was sometimes used to treat these patients, but lasting benefit was reported in few cases, ${ }^{16,19-22}$ and complications could be severe, including diplopia, hyperacusia, jaw deviation, corneal ulcers, corneal anesthesia, and anesthesia dolorosa. $^{16,19-22}$

\section{PATIENT SELECTION FOR DBS AND METHODOLOGICAL QUESTIONS}

\section{Definition of chronicity for surgical purposes}

The International Classification of Headache Disorders defines cluster headache as chronic when attacks occur for more than 1 year without remission or with remissions of less than 1 month; however, the criteria allow a diagnosis of chronic cluster headache when the patient has only three to four attacks per month over the preceding year. ${ }^{10}$ It is not prudent to select patients for an invasive surgical procedure such as DBS if they experience only three to four attacks per month. The first patients to undergo posterior hypothalamic implant had experienced severe attacks occurring many times daily without relief for periods of years. ${ }^{16}$ In subsequent studies using another experimental procedure (occipital nerve stimulation, which is less invasive), the criteria were not so strict, ${ }^{23}$ and some selected patients had only a few attacks per month. One result of the lack of standardized selection criteria is that patients from different series are not comparable in terms of illness severity. This problem is particularly acute when seeking to assess the efficacy of hypothalamic stimulation in comparison with occipital nerve stimulation.

We recommend that posterior hypothalamic stimulation be considered only for cluster headache patients who have experienced daily or almost attacks for least 1-2 years. $^{24}$

\section{Definition of drug resistance for surgical purposes}

Approximately $15-20 \%$ of cluster headache patients have the chronic form and need continuous medical care because of persistent pain. ${ }^{17,18}$ Clinical experience indicates that drug treatments can control or prevent attacks in approximately $80 \%$ of patients with chronic cluster headache. ${ }^{17,18}$ In some cases, drugs lose efficacy over time, often after years of successful pharmacologic control; less frequently, patients are unresponsive to medications from the outset. ${ }^{17,18}$

The term drug-resistant has been used to refer to patients who do not respond to one or more types of drug, ${ }^{25}$ as well as to those who do not respond to any of the known medications. ${ }^{24,26}$ Patients unresponsive to one or more types of drug may well respond to untried medi- 
cations, and should therefore be designated as partially drug-resistant, with the term completely drug-resistant confined to patients do not obtain pain prophylaxis with any medication. ${ }^{26}$ The condition of complete drug resistance is likely to be much more serious than that of partial resistance. Completely drug-resistant patients often resort to numerous medications (e.g., analgesics, triptans, nonsteroidal anti-inflammatory agents, opioids, ergot-derivatives, steroids) in increasingly desperate attempts to relieve their daily headaches. At our center, daily steroids and daily triptans are the drugs most frequently used by completely drug-resistant chronic cluster headache patients to abort ongoing attacks. Sumatriptan is the drug of choice, as it quickly blocks crises in most patients $;{ }^{27}$ however, sumatriptan resistance has emerged in some cases, with devastating effects when other drugs do not work. Some patients have injected sumatriptan (mean, $24 \mathrm{mg} / \mathrm{d}$ ) for years without adverse effects or electrocardiographic or other changes. ${ }^{28}$ It is recognized, however, that frequent triptan use may facilitate onset of myocardial ischemia in predisposed patients and may increase blood pressure.

Steroids are often dramatically successful in cluster headache; however, prolonged use of steroids (over years) may have fearful consequences, including chronic intestinal bleeding, bone demineralization leading to fractures and aseptic necrosis of the femoral head, fluid retention leading to heart failure, hypertension, bulimia, marked weight increase, severe myopathy with loss of ability to walk, and glaucoma. Agitation, insomnia, and even frank psychosis may also be effects of chronic steroid use. ${ }^{29}$

For these reasons, we have proposed that patients be designated as completely drug-resistant and considered for DBS only when all indicated drugs in the guidelines ${ }^{29}$ or in the wider literature have been tried (unless specifically contraindicated in a given case) at adequate dose and for a sufficient period but have nonetheless proved to be ineffective or associated with intolerable adverse effects. ${ }^{24,26}$

\section{Outcome measures for surgical purposes}

The first aim of treatment should be pain reduction. Clinical experience is that chronic cluster headache patients above all request a reduction in the number of attacks (which typically occur daily). In line with this experience, the International Headache Society (IHS) guidelines propose that reduction in number of daily attacks should by the main aim of prophylactic treatment. ${ }^{30}$ Other important endpoints are pain intensity and duration of attack. ${ }^{30}$ A patient's expressed satisfaction with the overall treatment should also be considered. In a pilot study on occipital nerve stimulation for cluster headache, attack frequency was unchanged in patients who nevertheless said they were satisfied with the treatment and would recommend it to other patients with cluster headache. ${ }^{31}$ This finding suggests that factors other than pain have a role in patient assessment of treatment; it further suggests that patient satisfaction should not be a primary endpoint for studies assessing the efficacy of hypothalamic or occipital nerve stimulation in cluster headache. ${ }^{32}$ The IHS guidelines recommend that treatment efficacy should be monitored by getting patients to report the number of attacks per day in a headache diary. ${ }^{30}$ To this we add that patients should also be asked to report pain intensity (on a visual analog scale) and attack duration (in minutes), particularly when patients undergoing hypothalamic stimulation are being monitored. Monitoring all these indicators will reduce the possibility of bias in assessing efficacy.

\section{RESULTS OF HYPOTHALAMIC STIMULATION}

\section{Acute pain}

To evaluate a possible acute effect of hypothalamic stimulation on cluster headache attacks, 136 attacks were acutely treated in 16 patients who had received hypothalamic implants to prevent chronic cluster headache. ${ }^{33}$ In the immediate postoperative period, when the stimulator was off and headache attacks were frequent, the stimulator was turned on in bipolar mode as soon as an attack began. Starting at 7-14 days after implantation, the stimulator was on in monopolar mode and amplitude was slowly increased to achieve prophylaxis. Patients were still experiencing headache attacks, and these were acutely treated in monopolar mode by abruptly increasing the amplitude as soon as an attack occurred. Stimulation frequency was $180 \mathrm{~Hz}$ and pulse width was $60 \mu \mathrm{s}$, because these were the settings effective in preventing chronic cluster headache. The maximum stimulation amplitude tolerated was usually $4-5 \mathrm{~V}$. Patients could request acute stimulation termination at any moment, and rescue medication was available. Pain intensity was reported on a visual analog scale. Blood pressure, heart rate, sweating, and other vital and neurological signs were monitored. For the 108 attacks in 16 patients, 23\% of the pain attacks improved (i.e., intensity was reduced by $>50 \%$ ), but only $16 \%$ disappeared completely during acute stimulation. From these data, it is evident that acute hypothalamic stimulation is ineffective as a treatment for cluster headache. ${ }^{33}$ This is consistent with experience in the prevention of chronic cluster headache: typically, several weeks of continuous stimulation are required before attacks are markedly reduced or eliminated. ${ }^{16,34-45}$

\section{Chronic pain}

Long-term hypothalamic stimulation was first tried in a patient in whom further lesional surgery was contraindicated; exceptional benefit was achieved, with no major adverse effects. ${ }^{16}$ The same group subsequently reported on 16 drug-resistant chronic cluster headache patients 
TABLE 1. Reported Improvement with Hypothalamic Stimulation for Drug-Resistant Chronic Cluster Headache Patients

\begin{tabular}{|c|c|c|c|}
\hline References & Patients Implanted, no. & Mean Follow-Up, yr & Patients Improved, $*$ no. $(\%)$ \\
\hline Schoenen et al., ${ }^{37} 2005$ & 4 & 4 & $2(50)$ \\
\hline Benabid et al., ${ }^{43} 2006^{*}$ & 1 & 1 & $1(100)$ \\
\hline D'Andrea et al., ${ }^{42} 2006^{*}$ & 3 & 2.5 & $2(66)$ \\
\hline Leone et al., ${ }^{38} 2006^{\dagger}$ & 16 & 4 & $10(62)$ \\
\hline Black et al. ${ }^{48} 2007^{*}$ & 2 & 2.6 & $2(100)$ \\
\hline Mateos et al., ${ }^{44} 2007^{*}$ & 2 & 1 & $2(100)$ \\
\hline Owen et al., ${ }^{39}$ 2007; Brittain et al., ${ }^{47} 2009$ & 3 & 1 & $3(100)$ \\
\hline Starr et al., ${ }^{40} 2007$; Sillay et al., ${ }^{46} 2010$ & 8 & 1 & $5(62)$ \\
\hline Bartsch et al., ${ }^{41} 2008$ & 6 & 1.4 & $3(50)$ \\
\hline Fontaine et al., 452010 & 11 & $>1$ & $6(55)$ \\
\hline Nikka et al. ${ }^{\ddagger}$ & 2 & 2 & $0(0)$ \\
\hline Total & 58 & - & $36(62)$ \\
\hline
\end{tabular}

Improvement was defined as pain free or almost pain free.

*Presented in abstract form only.

${ }^{\dagger}$ See text for other findings midway through and after the overall 4-year follow-up.

${ }^{\ddagger}$ Personal communication. Data reported with permission of the authors (Nikkhah G, personal communication, 2007).

who received hypothalamic implants after a mean follow-up of more than 4 years (Table 1$){ }^{38}$ After the first 2 years, pain abolition or major pain reduction was obtained in 13 of the 16 patients (and in 15 of 18 implants, or $83 \%)$. After 4 years, a persistent pain-free state was still present in 10 patients (62\%), although 4 of the patients also required additional medical prophylaxis to control the attacks. ${ }^{38}$ The hypothalamic stimulation subsequently became ineffective in 3 of the 10 patients, notwithstanding many changes in stimulation settings; however the illness also changed from chronic to episodic in the same 3 patients, and they experienced months of complete remission punctuated by periods of brief but typical attacks.

Similar improvements have been reported from other studies. Over 11 studies, 36 of 58 patients $(62 \%)$ obtained improvement, defined as pain free or almost pain free (Table 1).

Fontaine et al. ${ }^{45}$ performed a double-blind prospective crossover study on 11 patients recruited from centers throughout France, to assess the efficacy and safety of unilateral hypothalamic stimulation for severe chronic drug-resistant cluster headache. The randomized phase compared active with sham stimulation over 1-month periods, and was followed by a 1-year open phase. The primary outcome was weekly attack frequency. Other outcomes were pain intensity, number of sumatriptan injections, and changes in emotional impact and quality of life, as assessed by the Hospital Anxiety and Depression Scale and the SF-12 Health Survey, respectively. Tolerance was assessed by active surveillance of behavior and hormone levels. During the randomized phase, there was no significant difference in primary or secondary outcome measures between active and sham stimulation. At the end of the open phase, chronic stimulation had reduced weekly attack frequency by more than $50 \%$ in 6 of the 11 patients, 3 of whom were pain-free. There were three serious adverse events: subcutaneous infection, transient loss of consciousness, and micturition syncope. There were no significant changes in hormonal function or electrolytic balance. Although the randomized phase showed no efficacy of hypothalamic stimulation, the open phase indicated long-term efficacy in more than $50 \%$ of patients without high morbidity.

In a study in California, five of the eight cluster headache patients with hypothalamic implants obtained greater than $50 \%$ reduction in headache intensity or frequency after at least 1 year, although none were completely pain free..$^{40,46}$ In a multicenter study in Germany, three of the six cluster headache patients with hypothalamic implants achieved almost pain-free status, with treatment failure in the other three. ${ }^{41}$ In a study from a Belgian center, two of the four patients with hypothalamic implants were pain free and one had improved substantially. ${ }^{37}$ In a study from Oxford, UK, all three patients with hypothalamic implants were reported as pain free and with no adverse effects after a follow-up of 6 months to 2 years. ${ }^{39,47}$ Other outcomes from studies presented only in abstract form are included in Table $1,{ }^{42-44,48}$ along with data from one unpublished study.

\section{Tolerability and adverse events}

As with any cerebral electrode implantation procedure, implantation to the hypothalamus is associated with a small risk of intracerebral hemorrhage and must be performed by an expert neurosurgical team, and in patients without specific risk factors for cerebral hemorrhage. ${ }^{38}$ Investigators at the center with the largest experience (16 patients were given 18 implants) reported a small transient nonsymptomatic hemorrhage into the third ventricle in 1 case, identified on postoperative CT; follow-up 
CT revealed complete resolution. ${ }^{38}$ No untoward intraoperative modifications in EEG, blood pressure, temperature, breathing, affective state, or state of consciousness were observed. ${ }^{38}$

In one study, one of the six patients implanted died soon after the operation, because of implantation-induced intracerebral hemorrhage; in another patient, the implantation was stopped because of panic attack. ${ }^{37}$ It is possible that this patient had anxiety disorder; alternatively, the electrode may have missed the target. Panic attacks have been reported during periaqueductal stimulation, ${ }^{3}$ but not during hypothalamic implantation or stimulation. $^{16,34-36,38-48}$

Visual disturbances, mainly diplopia, are the primary limiting adverse effect induced by hypothalamic stimulation. ${ }^{16,34-45}$ Diplopia occurs in most patients when the amplitude is increased too rapidly; however, it subsides within a few minutes, although a few days may be required for complete disappearance. ${ }^{16,34-45}$ No changes in electrolyte balance, body temperature, blood pressure, sleep-wake cycle, appetite or thirst, or EEG findings have been observed on prolonged hypothalamic stimulation. ${ }^{16,34-45}$ Levels of cortisol, prolactin, thyroid hormones, thyroid-stimulating hormone, and testosterone also remain unchanged. ${ }^{16,34-45}$ Weight loss of approximately $3 \mathrm{~kg}$ generally occurs in the first 3-6 postoperative months, which may be due to steroid withdrawal, although a central effect of neurostimulation cannot be completely ruled out. ${ }^{38}$

One patient ceased menstruating 4 months before implantation as a result of excessive drug use (both therapeutic and over the counter); her cycles returned to normal 1 month after implantation. ${ }^{38}$ Neither depressive or dysphoric symptoms nor changes in character or behavior have been observed. ${ }^{16,34-45}$ In the study of Fontaine et al., ${ }^{45}$ a double-blind multicenter study involving 11 patients, no significant changes in hormone levels or electrolytic balance were observed, but subcutaneous infection, transient loss of consciousness, and micturition syncope were major adverse effects.

\section{THE AUTONOMIC NERVOUS SYSTEM DURING HYPOTHALAMIC STIMULATION}

The human hypothalamus occupies approximately 4 $\mathrm{cm}^{3}$, or $0.3 \%$ of total brain volume, ${ }^{49}$ and yet contains integrator systems that, via the autonomic and endocrine effector systems, regulate fluid and electrolyte balance, food ingestion and energy balance, reproductive functions, thermoregulation, immune responses, and many emotional responses, including pain. Many of the human hypothalamic nuclei appear to have largely conserved anatomy and function, compared with lower animals. ${ }^{49,50}$ Parasympathetic effects predominate when the anterior hypothalamus is stimulated, and sympathetic ef- fects tend to occur when the posterior hypothalamus is stimulated. ${ }^{49}$ Lesions of the ventromedial nucleus cause increased vagal and decreased sympathetic tone; the paraventricular nucleus is an important autonomic integrative center. ${ }^{51}$ This does not, however, imply the existence of discrete parasympathetic or sympathetic centers. Stimulation to disparate parts of the hypothalamus can cause profound changes in heart rate, cardiac output, vasomotor tone, peripheral resistance, differential blood flow in organs and limbs, frequency and depth of respiration, alimentary tract motility and secretion, and erection and ejaculation. ${ }^{49}$

With the development of hypothalamic stimulation, the human hypothalamus has for the first time received long-term stimulation, ${ }^{16}$ providing an opportunity to study this brain area. Cortelli et al. ${ }^{52}$ investigated aspects of autonomic nervous system function in eight patients with chronic drug-resistant cluster headache, before and after implantation of an electrode for unipolar stimulation of the posterior hypothalamus. Systolic and diastolic blood pressure, cardiac output, total peripheral resistance, heart rate, and oronasal and abdominal breathing were monitored continuously at supine rest, during the head-up tilt test (HUTT), Valsalva maneuver, deep breathing, the cold face test, isometric handgrip, and baroreflex. The results before and after surgery were compared. Continuous long-term unipolar stimulation was associated with an enhanced sympatho-excitatory effect on the cardiovascular system during HUTT, whereas the Valsalva maneuver, deep breathing, cold face test, isometric handgrip, and baroreflex sensitivity were unaffected. These findings suggest that chronic stimulation of the posterior hypothalamus can alter the modulation of orthostatic adaptation without affecting the baroreflex, cardiorespiratory interactions, or efferent sympathetic and vagal functions. ${ }^{52}$ Thus, in the supine resting position, the cardiovascular system is not influenced by posterior hypothalamic stimulation, providing important evidence for the safety of long-term stimulation. $^{52}$

Posterior hypothalamic stimulation in animals produces marked changes in autonomic functions. ${ }^{53}$ In humans, continuous long-term posterior hypothalamic stimulation induces fewer and less marked changes than in animals, probably because in humans the stimulation is of lower amplitude and is increased more slowly than in animals.

\section{Sleep and circadian rhythm of body core temperature during posterior hypothalamic stimulation}

The hypothalamus, especially its posterior part, is involved in control of the sleep-wake cycle and regulation of arousal. ${ }^{54}$ Body core temperature and the structure and quality of sleep were investigated in three chronic drug- 
resistant cluster headache patients before and during chronic lasting stimulation of the posterior hypothalamus. ${ }^{55}$ The patients underwent 48 hours of consecutive polysomnography (PSG), using the Vitaport system (Temec Instruments, Kerkrade, Netherlands; for details, see Starr et $\mathrm{al}^{40}{ }^{4}$ ), with body core temperature monitored by rectal probe. In these patients, nocturnal cluster headaches disappeared during hypothalamic stimulation, although in two patients PSG revealed that occasional daytime attacks were occurring 4 months after implantation. Polysomnography during stimulation revealed more continuous sleep, with increased total sleep time and sleep efficiency, with slow-wave sleep stages becoming more prominent; indices of fragmented sleep (arousals, periodic limb movements during sleep [PLMS], and stage shift indices) were reduced. Thus, posterior hypothalamic stimulation was associated with improved sleep structure and quality, compared with the preoperative condition. ${ }^{55}$ It is likely that the main cause of sleep improvement was the disappearance of nocturnal headache attacks. Nevertheless, the reduction in PLMS suggested a direct effect of stimulation. Because PLMS were reduced in light sleep as well as in non-REM sleep, the reduction cannot be attributed simply to the reduced time spent in light sleep. No daytime sleepiness or sleep-related breathing disorders were observed with posterior hypothalamic stimulation, and body core temperature rhythm was also normal, providing further evidence of the safety of hypothalamic stimulation. ${ }^{55}$

\section{Microrecordings}

Hypothalamic stimulation has provided an opportunity to use microrecording techniques to investigate the characteristics of the neurons at that level. Microrecording data from the stereotactic target site are discussed in what follows. This area does not correspond to a specific anatomical entity, and there is no consensus as to whether it is part of the posterior hypothalamus or anterior periventricular gray matter. ${ }^{56}$ Two sets of stereotactic coordinates have been used to identify this target, those published by Leone et al. ${ }^{16}$ in $2001(x=2 \mathrm{~mm}$ lateral to the midline, $y=6 \mathrm{~mm}$ behind the midcommissural point, $z=8$ below the commissural plane) and the revised coordinates published by Franzini et al. ${ }^{34}$ in 2003 $(x=2 \mathrm{~mm}$ lateral, $y=3 \mathrm{~mm}$ posterior, and $5 \mathrm{~mm}$ inferior to the midcommissural point).

Several studies have obtained single-unit microrecordings from this region in cluster headache patients. ${ }^{34,37,40,41,46,57,58}$ In all but one study, ${ }^{47}$ the microrecordings were obtained from patients not experiencing an attack (i.e., under basal conditions). Franzini et al. ${ }^{34}$ used a Medtronic (Minneapolis, MN) lead point system to investigate the target area. They presented a figure illustrating electrical activity in a single unit, but provided no quantitative data. Schoenen et al. ${ }^{37}$ obtained data from the area defined by the stereotactic coordinates of Leone et al. ${ }^{16}$ in four chronic cluster headache patients. They used a Medtronic 9013-SD-08411 microelectrode and Medtronic lead point system, starting $10 \mathrm{~mm}$ above the target and continuing at $1-\mathrm{mm}$ intervals down to the target. ${ }^{37}$ No additional procedural details were given, and no quantitative data. No specific neuronal activity — similar to that found in DBS of the subthalamic nucleus and globus palliduswas found, but in two patients bursts of action potentials synchronous with heart beats were recorded. ${ }^{37}$

Cordella et al. ${ }^{57}$ reported quantitative microrecording data obtained with the Medtronic lead point system from one patient with cluster headache and one patient with SUNCT. The target coordinates were those of Franzini et al. ${ }^{34}$ Patients were studied fully awake. Recording began as the microelectrode reached the hypothalamic target. Signals from a single cell in each patient were collected for sufficient time to permit offline analysis with the CED Spike2 package (Cambridge Electronic Design, Cambridge, UK). Average firing rate was 24 per second. Most recordings showed isolated action potentials, and most interstimulus intervals were in the range of 10-15 ms. Autocorrelograms of the two cells showed no periodicity of discharge rate, but a $1 \mathrm{~Hz}$ oscillatory pattern was identified in the SUNCT patient, possibly a pulse artefact. ${ }^{57}$

A California group ${ }^{40,58}$ implanted an electrode in the posterior hypothalamus of cluster headache patients, using the coordinates of Franzini et al. ${ }^{34}$ Single microelectrode penetration was performed beginning $10 \mathrm{~mm}$ above the anatomic target, without systemic sedation. Six patients and 24 units were evaluated. Individual units were discriminated offline by cluster cutting in principal-component space. Discharge rates, interspike intervals, and oscillatory activity were analyzed and compared with activity in the ventromedial thalamic nucleus of the same patient. The posterior hypothalamic neurons investigated were characterized by slow, regular spontaneous discharge with wide, low-amplitude action potentials. The mean discharge rate of the posterior hypothalamic units was significantly lower (mean, $\sim 13 \mathrm{~Hz}$ ) than that from thalamic units (mean, $28 \mathrm{~Hz}$ ). Oscillatory activity was not detected. There was no morbidity. ${ }^{40,58}$

Bartsch et al. ${ }^{41}$ reported on four patients who received posterior hypothalamic implants for drug-resistant cluster headache. Microrecordings were obtained at $1 \mathrm{~mm}$ around the target (coordinates of Franzini et al. ${ }^{34}$ ). In three patients, sensory stimulation of the trigeminal and spinal dermatomes was performed and in two patients, motor stimulation (passive movement of elbow), autonomic testing (counting backwards under stress, cold pack, and bladder filling), and affective stimulation (pictures with emotional content) were also performed. Units had a tonic, nonrhythmic firing pattern with an average discharge rate of $17 \mathrm{~Hz}$ (range, 13-35). None of the 
applied stimuli produced an obvious response. Microrecordings were obtained using high-impedance microelectrodes, making it possible to pick up discharges from a single neuron. ${ }^{41}$

It is evident from the literature that it is not easy to obtain consistent microelectrode recordings from this area of the brain. Both drugs and level of vigilance can affect the data obtained. Sani et al. ${ }^{58}$ suggested that abnormal impedance or lack of activity indicates that the exploring microelectrode may be in the interpeduncular cistern, ${ }^{30}$ and pulsation artefact is frequent. A discharge rate of approximately $20 \mathrm{~Hz}$ without specific rhythmic pattern could be the neurophysiological hallmark of neurons in this area in cluster headache patients, using both the coordinates of Leone et al. ${ }^{16}$ and of Franzini et al. ${ }^{34}$

The possibility remains that neuronal activity in this region differs in other conditions, and perhaps even in cluster headache patients during an attack. Brittain et al. ${ }^{47}$ recorded local field potentials during a cluster headache attack that occurred during surgical implantation of a hypothalamic electrode. Local field potentials were characterized by a significant increase in power during the attack. ${ }^{47}$ This appears to be the first report of neuronal activity during a cluster headache and lends strong support to data (mainly from use of indirect hemodynamic neuroimaging techniques) implicating hypothalamic activation in cluster headache generation.

\section{Pain threshold}

Although stimulation of the posterior hypothalamus is now established as a clinically effective means of preventing headache attacks in chronic cluster headache patients, the underlying mechanisms are not well understood. The hypothalamus is known to be connected to the trigeminal system via the trigeminohypothalamic tract. ${ }^{59,60}$ In an investigation of whether hypothalamic stimulation affects heat and pain perception in the first trigeminal branch, Jürgens et al. ${ }^{61}$ examined chronic cluster headache patients with an electrode implanted unilaterally in the posterior hypothalamus $(n=11)$, chronic cluster headache patients with no implanted electrode $(n=15)$, and healthy controls $(n=29)$. Perception and pain thresholds for hot and cold stimuli were determined bilaterally at the forearm, at the lower leg, and supraorbitally. Cold pain thresholds in the distribution of the first trigeminal branch were significantly higher on the stimulated side of implanted patients, compared with healthy controls. ${ }^{59}$ Stimulated implanted patients also had significantly higher cold detection thresholds than did nonimplanted cluster headache patients. Short-term interruption of stimulation did not change thresholds. ${ }^{59}$ These findings support the notion that neurostimulation of the posterior hypothalamus in cluster headache affects only certain aspects of pain sensation. ${ }^{59}$

\section{SUNCT SYNDROME AND PAROXYSMAL HEMICRANIA}

The SUNCT syndrome is often refractory to medication, ${ }^{62}$ and severely affected patients may consent to surgical ablation of the trigeminal nerve, even though outcomes are generally poor. ${ }^{63}$ After a functional MRI study had shown increased blood flow in the ipsilateral posterior inferior hypothalamus during SUNCT attacks, ${ }^{12}$ the posterior inferior hypothalamus of a patient experiencing severe intractable SUNCT was implanted. ${ }^{64}$ Prolonged stimulation resulted in long-lasting pain relief without continuous drug administration and with virtually no adverse effects. ${ }^{64}$ When the stimulator was turned off, without the patient's knowledge, the crises reappeared but gradually disappeared after it was turned back on. ${ }^{64}$ Another drug-resistant SUNCT patient received ipsilateral hypothalamic implantation: the patient experienced an immediate $63 \%$ decrease in the mean number of attacks per day, and a progressive decline in attack frequency; at 12 months after implantation, daily attack frequency had stably reduced by $80 \%$, relative to preoperative frequency. ${ }^{65}$

Paroxysmal hemicrania is a rare trigeminal autonomic cephalalgia characterized by severe, unilateral, orbitalsupraorbital, or temporal pain attacks lasting 2-30 minutes and accompanied by ipsilateral craniofacial autonomic manifestations. ${ }^{10}$ Attacks usually occur several times daily, and are completely prevented by adequate doses of indomethacin. ${ }^{10}$ Nonetheless, some patients cannot tolerate the adverse effects of indomethacin and may therefore be candidates for surgery. The therapeutic effectiveness of ipsilateral-to-the-pain posterior hypothalamic stimulation was assessed in a patient with $\mathrm{PH}$ intolerant to medical management. ${ }^{66}$ The patient's headache improved markedly soon after stimulation was started, and no complications were observed. On two separate occasions, the stimulator was switched off and the attacks reappeared within minutes.

It is noteworthy that a previous PET study found significant activation of the contralateral posterior hypothalamus in untreated $\mathrm{PH},{ }^{13}$ whereas ipsilateral posterior hypothalamic activation has been demonstrated during a cluster headache attack. ${ }^{11}$ Nevertheless, ipsilateral hypothalamic stimulation was effective in the patient with $\mathrm{PH}$, as already noted ${ }^{64}$ just as it is in cluster headache. ${ }^{16,34-45}$ In the patient with $\mathrm{PH}$, stimulation was effective in controlling attacks within minutes, ${ }^{64}$ whereas several weeks of stimulation are necessary to gradually bring cluster headache attacks under control. ${ }^{16,34-45}$ Finally, note that before implantation the attacks in this $\mathrm{PH}$ patient lasted 2-3 minutes, ${ }^{64}$ which is at the short extreme of attack duration for this headache, but typical of the duration for SUNCT. 


\section{CONCLUSIONS}

Ten years after its introduction, ${ }^{16}$ the efficacy of hypothalamic stimulation as a treatment for chronic drugresistant cluster headache can be considered established. ${ }^{34-45}$ Hypothalamic stimulation has generally been performed safely, but in view of the invasiveness of the procedure, should be offered only to patients with chronic and completely drug-resistant cluster headache (or other trigeminal autonomic cephalalgia) who experience multiple attacks a day. ${ }^{34-45}$ Hypothalamic stimulation takes time to exert its therapeutic effect in cluster headache, and frequent adjustment of stimulation variables is required to maintain optimal benefit. ${ }^{34-45} \mathrm{Neu}-$ roimaging investigations have shown that hypothalamic stimulation activates a number of brain areas pertaining to the pain matrix and deactivates others. Hypothalamic stimulation activates the ipsilateral trigeminal complex,${ }^{67}$ but this does not cause the patient to perceive disturbances within the trigeminal distribution. This finding clearly indicates that simultaneous activation of the hypothalamus and of the trigeminal system are both necessary, but not sufficient, to generate cluster headache attacks. In addition to the hypothalamus, therefore, other as yet unknown brain areas are likely to play a primary role in the pathophysiology of cluster headache and of other trigeminal autonomic cephalalgias. ${ }^{68}$

\section{REFERENCES}

1. Gildenberg PL. The history of stereotactic and functional neurosurgery. In: Gildenberg PL, Tasker RR, editors. Textbook of stereotactic and functional neurosurgery. 1st ed. New York: McGraw Hill, 1998:5-20.

2. Bittar RG, Kar-Purkayastha I, Owen SL, Bear RE, Green A, Wang $S$, et al. Deep brain stimulation for pain relief: a meta-analysis. J Clin Neurosci 2005;12:515-519.

3. Veloso F, Kumar K, Toth C. Headache secondary to deep brain implantation. Headache 1998;38:507-515.

4. Young RF, Chambi VI. Pain relief by electrical stimulation of the periaqueductal and periventricular gray matter: evidence for a nonopioid mechanism. J Neurosurg 1987;66:364-371.

5. Sano K, Sekino H, Hashimoto I, Amano K, Sugiyama H. Posteromedial hypothalamotomy in the treatment of tractable pain. Confin Neurol 1975;37:285-290.

6. Raskin NH, Hosobuchi Y, Lamb S. Headache may arise from perturbation of brain. Headache 1987;27:416-420.

7. Bittar RG, Nandi D, Carter H, Aziz TZ. Somatotopic organization of the human periventricular gray matter. J Clin Neurosci 2005; $12: 240-241$.

8. Kumar K, Toth C, Nath R. Deep brain stimulation for intractable pain: a 15-year experience. Neurosurgery 1997;40:736-746.

9. May A. New insights into headache: an update on functional and structural imaging findings. Nat Rev Neurol 2009;5:199-209.

10. Headache Classification Committee of the International Headache Society. The International Classification of Headache Disorders, 2nd edition. Cephalalgia 2004;24(Suppl 1):8-160.

11. May A, Bahra A, Büchel C, Frackowiak RS, Goadsby PJ. Hypothalamic activation in cluster headache attacks. Lancet 1998;352: 275-278.

12. May A, Bahra A, Büchel C, Turner R, Goadsby PJ. Functional MRI in spontaneous attacks of SUNCT: short-lasting neuralgiform headache with conjunctival injection and tearing. Ann Neurol 1999;46:791-794.
13. Matharu MS, Cohen AS, Frackowiak RSJ, Goadsby PJ. Posterior hypothalamic activation in paroxysmal hemicrania. Ann Neurol 2006;59:535-545.

14. Matharu MS, Cohen AS, McGonigle DJ, Ward N, Frackowiak RS, Goadsby PJ. Posterior hypothalamic and brainstem activation in hemicrania continua. Headache 2004;44:747-761.

15. May A, Ashburner J, Büchel C, McGonigle DJ, Friston KJ, Frackowiak RS, et al. Correlation between structural and functional changes in brain in an idiopathic headache syndrome. Nat Med 1999;5:836-838

16. Leone M, Franzini A, Bussone G. Stereotactic stimulation of posterior hypothalamic gray matter for intractable cluster headache. N Engl J Med 2001;345:1428-1429.

17. Kudrow L. Cluster headache, mechanism and management. New York: Oxford University Press, 1980.

18. Sjaastad O. Cluster headache syndrome. Major Problems in Neurology 23. London, Philadelphia, Toronto: WB Saunders, 1992.

19. Jarrar RG, Black DF, Dodick DW, Davis DH. Outcome of trigeminal nerve section in the treatment of chronic cluster headache. Neurology 2003;60:1360-1362.

20. O'Brien MD, Kirkpatrick PJ, McCabe JJ. Trigeminal nerve section for chronic migrainous neuralgia. In: Olesen J, Goadsby PJ, editors. Cluster headache and related conditions. Frontiers in Headache Research 9. Oxford: Oxford University Press, 1999:291-295.

21. Donnet A, Valade D, Regis J. Gamma knife treatment for refractory cluster headache: prospective open trial. J Neurol Neurosurg Psychiatry 2005;76:218-221.

22. Matharu MS, Goadsby PJ. Persistence of attacks of cluster headache after trigeminal nerve root section. Brain 2002;125:976-984.

23. Magis D, Allena M, Bolla M, De Pasqua V, Remacle JM, Schoenen J. Occipital nerve stimulation for drug-resistant chronic cluster headache: a prospective pilot study. Lancet Neurol 2007; 6:314-321.

24. Leone M, May A, Franzini A, Broggi G, Dodick D, Rapoport A, et al. Deep brain stimulation for intractable chronic cluster headache: proposals for patient selection. Cephalalgia 2004;24:934937.

25. Goadsby PJ, Schoenen J, Ferrari MD, Silberstein SD, Dodick D Towards a definition of intractable headache for use in clinical practice and trials. Cephalalgia 2006;26:1168-1170.

26. Leone M, Proietti Cecchini A, Mea E, D'Amico D, Tullo V, Grazzi $\mathrm{L}$, et al. Therapeutic neurostimulation in chronic headaches: problems of patient selection. Neurol Sci 2008;29:S59-S61.

27. Sumaptriptan Cluster Headache Study Group. Treatment of acute cluster headache with sumatriptan. N Engl J Med 1991;325:322326.

28. Turhal NS. Sumatriptan overdose in episodic cluster headache: a case report of overuse without event. Cephalalgia 2001;21:700.

29. May A, Leone M, Áfra J, Linde M, Sándor PS, Evers S, et al. EFNS guidelines on the treatment of cluster headache and other trigeminal-autonomic cephalalgias. Eur J Neurol 2006;13:10661077.

30. Lipton RB, Micieli G, Russell D, Solomon S, Tfelt-Hansen P, Waldenlind E. Guidelines for controlled clinical trials of drugs in cluster headache. Cephalalgia 1995;15:452-462.

31. Burns B, Watkins L, Goadsby PJ. Treatment of medically intractable cluster headache by occipital nerve stimulation: long-term follow-up of eight patients. Lancet 2007;369:1099-1106.

32. Leone M, Franzini A, Cecchini AP, Broggi G, Bussone G. Stimulation of occipital nerve for drug-resistant chronic cluster headache. Lancet Neurol 2007;6:289-291.

33. Leone M, Franzini A, Broggi G, Mea E, Cecchini AP, Bussone G. Acute hypothalamic stimulation and ongoing cluster headache attacks. Neurology 2006;67:1844-1845.

34. Franzini A, Ferroli P, Leone M, Broggi G. Stimulation of the posterior hypothalamus for treatment of chronic intractable cluster headaches: first reported series. Neurosurgery 2003;52:1095-1099.

35. Franzini A, Ferroli P, Leone M, Bussone G, Broggi G. Hypothalamic deep brain stimulation for the treatment of chronic cluster headaches: a series report. Neuromodulation 2004;7:1-8.

36. Leone M, Franzini A, Broggi G, May A, Bussone G. Long-term follow up of bilateral hypothalamic stimulation for intractable cluster headache. Brain 2004;127:2259-2264. 
37. Schoenen J, Di Clemente L, Vandenheede M, Fumal A, De Pasqua $\mathrm{V}$, Mouchamps M, et al. Hypothalamic stimulation in chronic cluster headache: a pilot study of efficacy and mode of action. Brain 2005;128:940-947.

38. Leone M, Franzini A, Broggi G, Bussone G. Hypothalamic stimulation for intractable cluster headache: long-term experience. Neurology 2006;67:150-152.

39. Owen SLF, Green AL, Davies P, Stein JF, Aziz TZ, Behrens T, et al. Connectivity of an effective hypothalamic surgical target for cluster headache. J Clin Neurosci 2007;14:955-960.

40. Starr PA, Barbaro NM, Raskin NH, Ostrem JL. Chronic stimulation of the posterior hypothalamic region for cluster headache: technique and 1-year results in four patients. J Neurosurg 2007; 106:999-1005.

41. Bartsch T, Pinsker MO, Rasche D, Kinfe T, Hertel F, Diener HC, et al. Hypothalamic deep brain stimulation for cluster headache: experience from a new multicase series. Cephalalgia 2008;28:285295.

42. D'Andrea G, Nordera GP, Piacentino M. Effectiveness of hypothalamic stimulation in two patients affected by intractable chronic cluster headache. Neurology 2006;66(Suppl 2):A140 (abstract).

43. Benabid AL, Chabardès S, Seigneuret E, Torres N. Intraventricular stimulation for targets close to the midline: periaqueductal gray, posterior hypothalamus, anterior hypothalamus, subcommissural structures. Acta Neurochir (Wien) 2006;148:i-1xiv (abstract).

44. Mateos V, Seijo F, Lozano B, Álvarez Vega M, Fernández González F. Deep brain stimulation in refractory chronic cluster headache: first national cases [Estimulación cerebral profunda en cefalea en racimos crónica refractaria primeros casos nacionales] [In Spanish]. Neurologia 2007;22:96 (abstract).

45. Fontaine D, Lazorthes Y, Mertens P, Blond S, Géraud G, Fabre N, et al. Safety and efficacy of deep brain stimulation in refractory cluster headache: a randomized placebo-controlled double-blind trial followed by a 1-year open extension. J Headache Pain 2010; 11:23-31.

46. Sillay KA, Sani S, Starr PA. Deep brain stimulation for medically intractable cluster headache. Neurobiol Dis 2009 Jun 6 [Epub ahead of print].

47. Brittain J-S, Green AL, Jenkinson N, Ray NJ, Holland P, Stein JF, et al. Local field potentials reveal a distinctive neural signature of cluster headache in the hypothalamus. Cephalalgia 2009;29:11651173.

48. Black D, Bartleson JD, Torgrimson SM, Davis DH. Two cases of chronic cluster headache treated successfully with hypothalamic deep brain stimulation. Neurology 2007:P07.065 (abstract).

49. Swaab DF. The human hypothalamus: basic and clinical aspects; Part II: Neuropathology of the human hypothalamus and adjacent structures. Handbook of clinical neurology. Handbook of Clinical Neurology 80 (3rd series, vol. 2). Amsterdam: Elsevier, 20032004.

50. Sherrington CS. The integrative action of the nervous system. 2nd ed. New Haven, CT: Yale University Press, 1947:1.

51. Loewy AD. Forebrain nuclei involved in autonomic control. Prog Brain Res 1991;87:253-268.

52. Cortelli P, Guaraldi P, Leone M, Pierangeli G, Barletta G, Grimaldi D, et al. Effect of deep brain stimulation of the posterior hypothalamic area on the cardiovascular system in chronic cluster headache patients. Eur J Neurol 2007;14:1008-1015.

53. Benarroch EE. Homeostasis, immunomodulation and antinociception. In: Central autonomic network: functional organization and clinical correlations. Armonk, NY: Futura Publishing, 1997:405443.

54. Abrahamson EE, Moore RY. The posterior hypothalamic area: chemoarchitecture and afferent connections. Brain Res 2001;889: $1-22$.

55. Vetrugno R, Pierangeli G, Leone M, Bussone G, Franzini A, Brogli G, et al. Effect on sleep of posterior hypothalamus stimulation in cluster headache. Headache 2007;47:1085-1090.

56. Talairach J, Tournoux P. Co-planar stereotaxic atlas of the human brain: 3-dimensional proportional system: an approach to cerebral imaging. Stuttgart: G. Thieme Verlag, 1988.

57. Cordella R, Carella F, Leone M, Franzini A, Broggi G, Bussone G, et al. Spontaneous neuronal activity of the posterior hypothalamus in trigeminal autonomic cephalalgias. Neurol Sci 2007;28:93-95.

58. Sani S, Shimamoto S, Turner RS, Levesque N, Starr PA. Microelectrode recording in the posterior hypothalamic region in humans. Neurosurgery 2009;64(3 Suppl):161-167.

59. Malick A, Strassman AM, Burstein R. Trigeminohypothalamic and reticulohypothalamic tract neurons in the upper cervical spinal cord and caudal medulla of the rat. J Neurophysiol 2000;84:2078 2112.

60. Bartsch T, Levy MJ, Knight YE, Goadsby PJ. Differential modulation of nociceptive dural input to [hypocretin] orexin A and B receptor activation in the posterior hypothalamic area. Pain 2004; 109:367-378.

61. Jürgens T, Leone M, Proietti-Cecchini A, Busch V, Mea E, Bussone $\mathrm{G}$, et al. Hypothalamic deep-brain stimulation modulates thermal sensitivity and pain thresholds in cluster headache. Pain 2009;146:84-90.

62. Cohen AS, Matharu MS, Goadsby PJ. Short-lasting unilateral neuralgiform headache attacks with conjunctival injection and tearing (SUNCT) or cranial autonomic features (SUNA): a prospective clinical study of SUNCT and SUNA. Brain 2006;129:2746-2760.

63. Black DF, Dodick DW. Two cases of medically and surgically intractable SUNCT: a reason for caution and an argument for a central mechanism. Cephalalgia 2002;22:201-214.

64. Leone M, Franzini A, D’Andrea G, Broggi G, Casucci G, Bussone G. Deep brain stimulation to relieve severe drug-resistant SUNCT. Ann Neurol 2005;57:924-927.

65. Lyons MK, Dodick DW, Evidente VG. Responsiveness of shortlasting unilateral neuralgiform headache with conjunctival injection and tearing to hypothalamic deep brain stimulation. J Neurosurg 2009;110:279-281.

66. Walcott BP, Bamber NI, Anderson DE. Successful treatment of chronic paroxysmal hemicrania with posterior hypothalamic stimulation: technical case report. Neurosurgery 2009;65:E997.

67. May A, Leone M, Boecker H, Sprenger T, Juergens T, Bussone G, et al. Hypothalamic deep brain stimulation in positron emission tomography. J Neurosci 2006;26:3589-3593.

68. Leone M, Bussone G. Pathophysiology of trigeminal autonomic cephalalgias. Lancet Neurol 2009;8:755-764. 